\title{
Aberrantly hypermethylated tumor suppressor genes were identified in oral squamous cell carcinoma (OSCC)
}

Soo Yeon Kim¹, Yu Kyeong Han², Jae Min Song ${ }^{3,4}$, Chang Hun Lee ${ }^{5}$, Keunsoo Kang ${ }^{6}$, Joo Mi Yi ${ }^{2^{*+}}$ and Hae Ryoun Park ${ }^{1,4^{*}+}$

\begin{abstract}
Background: Oral squamous cell carcinoma (OSCC) is a genetic and epigenetic disease. There is growing evidence to suggest that environmental factors due to epigenetic changes can be involved in the OSCC pathogenesis. Although tumor suppressor genes (TSGS) are commonly inactivated by promoter hypermethylation in human cancers, the epigenetic changes and the mechanism of TSGs in human OSCC remain unclear. We therefore assessed the methylation status of the TSGs, which are associated with epigenetic silencing in human cancers, OSCC cell lines, primary tumors, and normal oral mucosa.

Results: We used 14 TSGs that were originally identified in colon cancer to investigate the aberrant hypermethylation of these genes associated with transcriptional silencing in 10 OSCC cell lines. We found three TSGS, TFPI2, SOX17, and GATA4, that are robustly hypermethylated and are associated with transcriptional silencing in OSCC cell lines. The re-expression of the three genes was induced by 5-aza-2'-deoxycytidine (5-aza-dC) in cells in which these genes were not expressed or had a lack of expression. In 33 cases of primary OSCC tumors, promoter hypermethylation was detected for the TFPI2, SOX17, and GATA4 genes at (32/33) 97\%, (22/33) 67\%, and (11/33) 33\%, respectively. Eleven normal oral mucosa samples showed no promoter hypermethylation for all three genes, which suggests that this promoter hypermethylation is cancer-specific. Bisulfite sequencing analysis confirmed the cancer-specific methylation of the TFPI2, SOX17, and GATA4 promoters in the OSCC cell lines and tumors but not in the normal oral mucosa samples. More importantly, the methylation status of TFPI2, GATA4, and SOX17 was significantly associated with OSCC patients' overall survival through TCGA DNA methylation database.
\end{abstract}

Conclusions: We identified that TFPI2, SOX17, and GATA4 are frequently hypermethylated in human OSCC cells in a cancer-specific manner and that the transcriptional expression of these genes is regulated by promoter hypermethylation in OSCC. Our results highlight the great potential used as a synergistic biomarker set to improve the prognosis and therapeutic treatment for patients with OSCC.

Keywords: DNA methylation, Tumor suppressor gene, Oral squamous cell carcinoma, Gene silencing, Prognosis biomarker

\footnotetext{
* Correspondence: jmyi76@inje.ac.kr; parkhr@pusan.ac.kr

† Joo Mi Yi and Hae Ryoun Park contributed equally to this work.

${ }^{2}$ Department of Microbiology and Immunology, College of Medicine, Inje

University, Busan 47392, Republic of Korea

${ }^{1}$ Department of Oral Pathology, School of Dentistry, Pusan National

University, Yangsan 50612, Gyeongsangnam-do, Republic of Korea

Full list of author information is available at the end of the article
}

(c) The Author(s). 2019 Open Access This article is distributed under the terms of the Creative Commons Attribution 4.0 International License (http://creativecommons.org/licenses/by/4.0/), which permits unrestricted use, distribution, and

reproduction in any medium, provided you give appropriate credit to the original author(s) and the source, provide a link to the Creative Commons license, and indicate if changes were made. The Creative Commons Public Domain Dedication waiver (http://creativecommons.org/publicdomain/zero/1.0/) applies to the data made available in this article, unless otherwise stated. 


\section{Background}

Epigenetic modification, including DNA methylation and many types of histone modifications, is responsible for the altered gene expression patterns that allow for specific phenotypes [1]. DNA methylation is the primary and most studied epigenetic modification [1] and plays an important role in normal mammalian development, but aberrant methylation patterns are correlated with several differentiation-related diseases, including many types of human cancers. Gene promoter hypermethylation in cancer can silence gene expression and regulate biological processes, especially tumor suppressor genes (TSGs), which have an important role in cancer initiation, progression, and metastasis [2]. Aberrant DNA methylation is a common phenomenon in malignancies, and the methylation profiles are altered in various tumors, which might be associated with clinical outcomes [1].

We and others have reported that the transcriptional silencing of tumor suppressor candidate genes is regulated by promoter hypermethylation in colorectal cancer $[3,4]$. High-throughput genome-wide methylation studies offer a sophisticated strategy to understand the significance of DNA methylation and its impact on gene regulation $[3,5]$.

Numerous studies have used these well-known tumor suppressor candidate genes in other cancer types, such as lung, breast, and pancreatic cancer types $[4,6]$. However, there is a lack of studies on the DNA methylation profiles of these TSGs in oral squamous carcinoma cancer (OSCC). Very recent findings suggest that the oral microbiota could contribute to CRC development, implying that the profile of epigenetic changes may be shared between two different cancer types $[7,8]$.

Oral squamous cell carcinoma (OSCC), the most prevalent type of head and neck SCC (HNSCC), typically behaves in an aggressive manner, frequently leading to local invasion and early lymph node metastasis [9]. The overall 5-year survival rate for OSCC is approximately $60 \%$ [10] and shows only modest improvement over the past two decades, despite considerable improvements in the treatment of OSCC $[11,12]$. In the last decade, there has been a lack of understanding of OSCC carcinogenesis, progression, and metastasis in terms of epigenetic regulation and its clinical application. Therefore, the identification of molecular changes for the oncogenes or TSGs associated with OSCC will help improve diagnosis predictions and early treatment $[13,14]$.

In this study, we investigated transcriptional silencing by the promoter hypermethylation of 18 tumor suppressor candidate genes (MGMT, sFRP2, HIC1, sFRP4, Timp3, sFRP5, TFPI2, p16, sFRP1, E-cad, SOX17, GATA4, GATA5, p14, FBN2, p15, and TCERG1L) in 10 OSCC cell lines and a small set of OSCC primary tumor samples $(n=33)$. Remarkably, we found that most of the genes are silenced by promoter hypermethylation in OSCC cell lines. Among these genes, TFPI2, SOX17, and GATA4 were frequently hypermethylated in both OSCC and oral cancer patient samples in a cancer-specific manner, suggesting that these genes may play a role as tumor suppressors in OSCC.

\section{Results \\ Identification of aberrantly hypermethylated tumor suppressor candidates in oral squamous cell carcinoma (OSCC)}

In this study, we investigated transcriptional silencing by the promoter hypermethylation of 14 well-known tumor suppressor candidate genes (MGMT, $s F R P 2, s F R P 1, H I C 1$, sFRP4, Timp3, sFRP5, TFPI2, p16, SOX17, GATA4, GATA5, $F B N 2$, and TCERG1L) in OSCC cell lines. These genes were originally identified from comprehensive studies of colon cancer as tumor suppressor candidate genes, and their transcriptional silencing is regulated by promoter hypermethylation in many other cancer types [4]. First, we examined whether the expression of these genes is regulated by promoter methylation in 10 OSCC cell lines (Ca922, HSC4, OSC20, SAS, SCC25, HN22, YD10B, YD38, YD9, and HSC3) by reverse transcriptase polymerase chain reaction (RT-PCR). We treated the OSCC cell lines with the demethylation agent 5-aza-2'-deoxycytidine (5-aza-dC) to determine whether these TSGs are re-expressed after 5aza- $\mathrm{dC}$ treatment. To correlate the transcriptional expression of TSGs with promoter hypermethylation, we assessed the methylation analysis of these TSGs in OSCC cell lines by methylation-specific PCR (MSP). We performed massive RT-PCR and MSP to identify whether these TSGs are silenced by promoter hypermethylation in OSCC. The results for transcriptional expression and promoter hypermethylation by the RT-PCR and MSP analyses of TSGs are summarized in Fig. 1a (Additional file 1: Figure S1) with the following three categories: (1) genes are silenced by promoter hypermethylation with re-expression by 5 -aza-dC treatment, (2) genes are silenced by promoter hypermethylation without re-expression by 5 -aza-dC treatment, and (3) gene expression is not correlated with promoter methylation. The methylation frequencies of 14 TSGs in 10 OSCC cell lines ranged from 50 to $100 \%$ (Fig. 1b), which suggests that all of the genes we tested are frequently hypermethylated in OSCC cell lines.

The silencing of most TSGs is regulated by promoter hypermethylation, and the genes are re-expressed by 5 aza-dC treatment. We found that the transcriptional silencing of 9 genes (MGMT, HIC1, sFRP4, Timp3, TFPI2, SOX17, GATA4, FBN2, and TCERG1L) out of 14 genes by promoter hypermethylation was correlated with gene re-expression by 5 -aza-dC treatment in 2 or more OSCC cell lines. Nonetheless, we decided to focus on 4 TSGs (TFPI2, SOX17, GATA4, and FBN2) because we later confirmed that these genes had a cancer-specific methylation pattern in primary OSCC tumor samples (Fig. 3). 


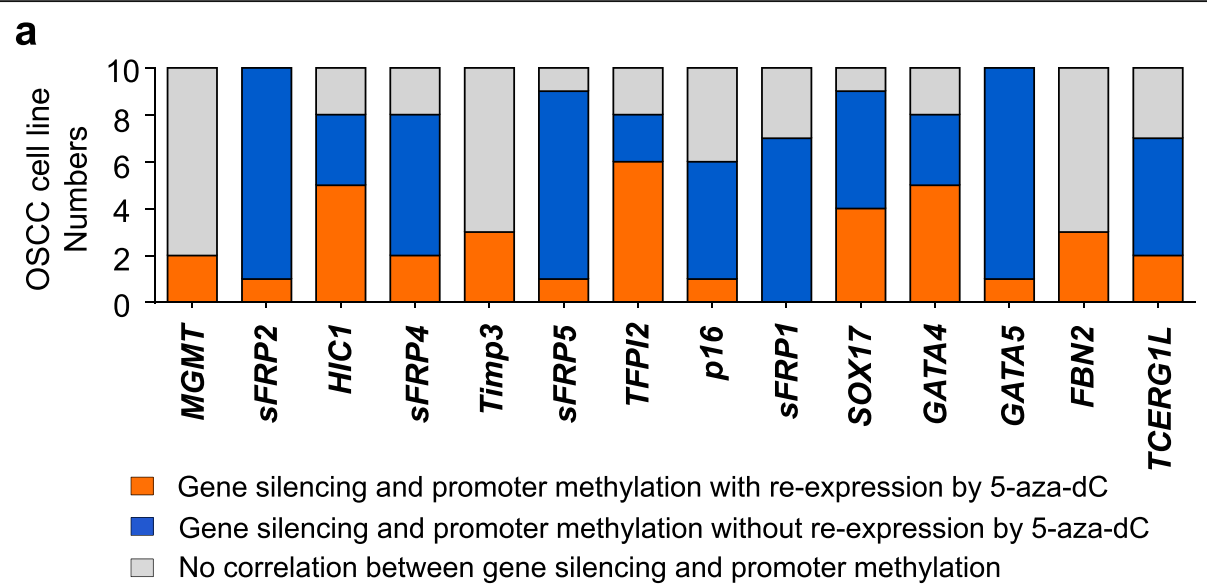

b

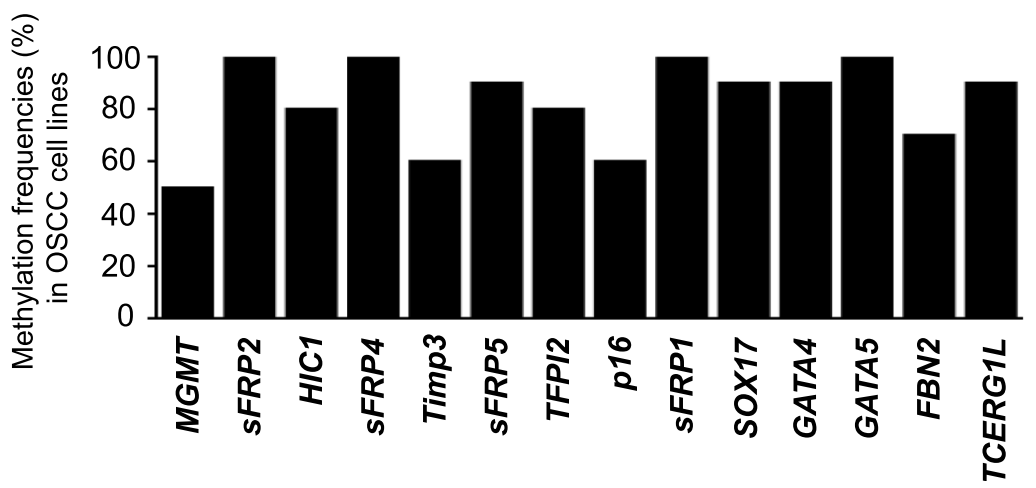

Fig. 1 Correlation between transcriptional expression and the promoter hypermethylation of tumor suppressor genes in OSCC cell lines. a Summarized results based on the RT-PCR and MSP results of 14 tumor suppressor genes in 10 OSCC cell lines (Ca9-22, HSC4, OSC20, SAS, SCC25, HN22, YD10B, YD38, YD9, and HSC3). The correlation between transcriptional expression and promoter hypermethylation was categorized by the following three criteria: (1) genes are silenced by promoter hypermethylation with re-expression by 5-aza-dC treatment (indicated in yellow), (2) genes are silenced by promoter hypermethylation without re-expression by 5 -aza-dC treatment (indicated in blue), and (3) genes are not correlated between gene expression and promoter methylation (indicated in gray). b Methylation frequencies of TSGs in 10 OSCC cell lines by MSP analysis

We determined whether these 4 genes are re-expressed after 5 -aza-dC treatment in 10 OSCC cell lines by realtime RT-PCR (Fig. 2). We postulate that these genes were re-expressed in OSCC cells after 5-aza-dC treatment, and we should detect their promoter hypermethylation in those cells. To verify that transcriptional silencing was due to promoter hypermethylation, we assessed the methylation levels of the TFPI2, SOX17, GATA4, and FBN2 genes by MSP. Overall, we identified four TSGs (TFPI2, SOX17, GATA4, and FBN2) that are regulated by promoter hypermethylation associated with transcriptional silencing in OSCC cell lines.

TFPI2, SOX17, and GATA4 are frequently hypermethylated in OSCC cells and primary OSCC tumors in a cancerspecific manner

To validate that four TSGs were indeed regulated by promoter hypermethylation, we extended our methylation analysis for these genes in a panel of primary OSCC tumor samples $(n=33)$ and normal oral mucosa samples $(n=11)$ obtained from cancer-free individuals. The clinical characteristics of the patients are documented in Table 1. Four genes (TFPI2, SOX17, GATA4, and FBN2) satisfied the criteria of "cancer-specific methylation" with high-frequency methylation in cell lines, no/undetectable methylation in normal oral mucosa (Fig. 3a), and frequent methylation in primary OSCC tumor samples (Fig. 3b). Based on our data, we decided to eliminate $F B N 2$ for further experimental analyses because of the low frequency $(<20 \%)$ of methylation in primary OSCC tissue samples.

We next assessed changes in DNA methylation status in the promoter region of the three genes by bisulfite genomic sequencing in representative OSCC cell lines (Ca9-22, HSC3, OSC-20, SAS, and YD10B), OSCC primary tumor samples $(n=5)$, and normal oral mucosa samples $(n=3)$ (Fig. 4). The location of promoter 
a

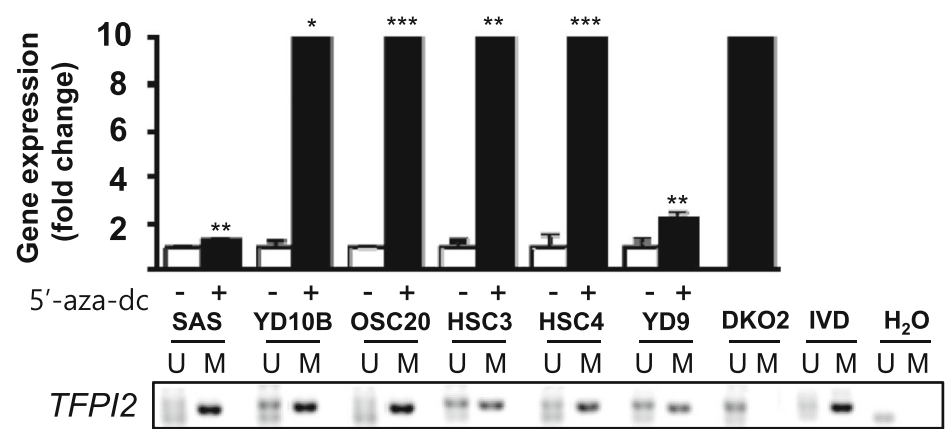

b

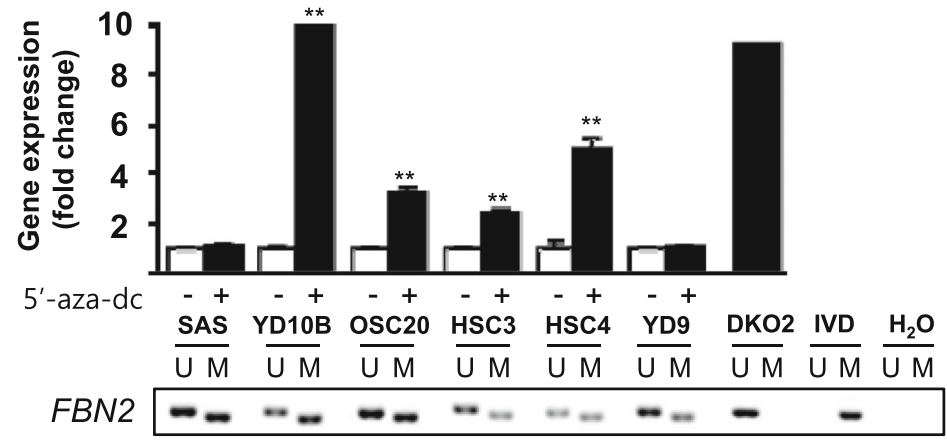

C

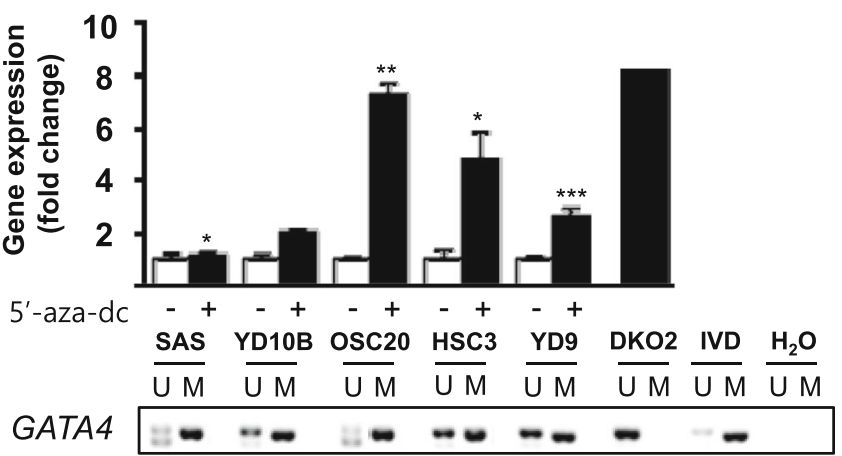

d

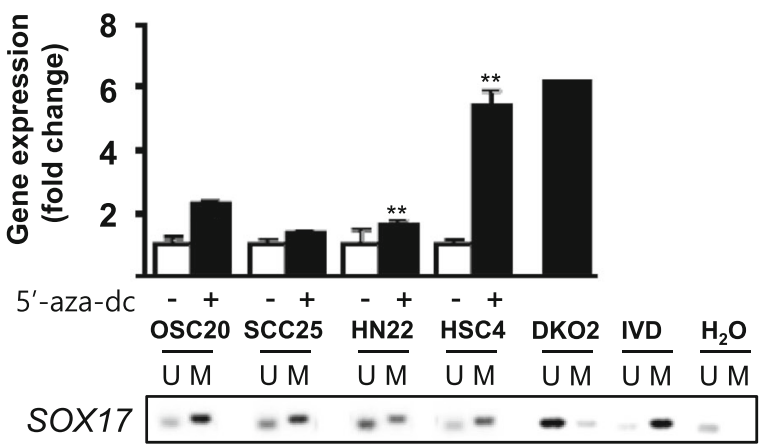

Fig. 2 a-d Epigenetic inactivation of TFPI2, SOX17, GATA4, and FBN2 in OSCC cell lines. The graph displays the expression analysis of four genes after 5-aza-dC treatment. Real-time RT-PCR analysis was carried out to assess four TSG expression levels in OSCC cell lines before and after treatment with $5 \mu \mathrm{M}$ 5-aza-dC for $72 \mathrm{~h}$. DNA methyltransferase 1 and $3 \mathrm{~b}$ knockout HCT116 cells (DKO) were included as positive controls. Asterisk indicates a significant increase in gene expression after 5 -aza-dC treatment $(p<0.05)$. The expression levels of genes were internally normalized to the expression levels of GAPDH, and the normalized expression for each gene before 5-aza-dC treatments was set to 1. Gel pictures describe the methylation analysis by methylation-specific PCR (MSP). PCR products recognize unmethylated (U) and methylated (M). DKO cells were used for the unmethylated control. IVD = in vitro methylated control; $\mathrm{ddH}_{2} \mathrm{O}=$ water control containing no DNA 
Table 1 Basic characteristics of the OSCC patient in this study

\begin{tabular}{ll}
\hline Characteristics & $N$ \\
\hline Total no. of patients & 34 \\
Age (years) & \\
Median (range) & $67(30-90)$ \\
Average (St. dev.) & $63.41(14.1)$ \\
Gender, $n$ (\%) & \\
Male & $18(52.9)$ \\
Female & $16(47.1)$ \\
Stage, $n$ (\%) & \\
1 & $8(23.5)$ \\
2 & $10(29.4)$ \\
3 & $4(11.8)$ \\
4 & $12(35.3)$ \\
Lymph node metastasis, $n$ (\%) & \\
Negative & $21(61.8)$ \\
Positive & $10(29.4)$ \\
Unknown & $3(8.8)$ \\
Grade, $n$ (\%) & $25(73.5)$ \\
Well differentiated & $9(26.5)$ \\
Moderate differentiated & $23(67.6)$ \\
Poor differentiated & $11(32.4)$ \\
No & \\
Nes & \\
\hline
\end{tabular}

regions (TFPI2: upstream region from -286 to $-76,30$ CpG sites; SOX17: region from +339 to +603 , 34 CpG sites; and GATA4: upstream region from -229 to 161 , $46 \mathrm{CpG}$ sites) for sequencing is relative to the transcription start sites (TSS) of exon 1. The bisulfite sequencing of five individual clones of PCR products from five OSCC cell lines revealed densely methylated CpG sites within the promoter regions of all of the clones. The methylation status of the TFPI2 promoter region in OSCC cells and primary tissues showed that most of the CpG sites (>99\%) we sequenced were methylated compared to those in the normal oral mucosal tissues $(<$ $11 \%)$. Both the SOX17 and GATA4 genes in OSCC cells and primary tissues (59-77\% for SOX17 and $71-91 \%$ for GATA4) showed relatively dense methylation than those in normal tissues $(<32 \%$ for SOX17 and $<23 \%$ for GATA4). Accordingly, these bisulfite sequencing data confirm the complete methylation of the three gene promoters in OSCC cell lines, primary tumor tissues, and normal oral mucosal samples determined by MSP (Fig. 2), but they also explain the lack of these three gene expression results in OSCC cell lines obtained by quantitative RT-PCR analysis. Notably, all three genes were significantly hypermethylated in primary OSCC tumors compared to those in the normal tissues. Overall, these results strongly suggest that the TFPI2, SOX17, and GATA4 genes showed increasing DNA methylation levels in primary OSCC tumors compared with the DNA methylation levels in normal oral tissues.

TFPI2, SOX17, and GATA4 protein expression is downregulated in primary OSCC tumor tissues

Next, we examined the protein expression of the three genes by immunohistochemical staining. Normal oral mucosal tissue showed strong nuclear positivity against TFPI2, SOX17, and GATA4 as well as cytoplasmic staining for TFPI2 and SOX17 (Fig. 5, Additional file 1: Figure S2). In contrast, those proteins were negatively expressed in both the nucleus and cytoplasm of the primary OSCC tissue.

\section{Methylation of TFPI2, SOX17, and GATA4 is associated with OSCC patient survival}

To investigate whether the methylation status of the three potential biomarkers (TFPI2, SOX17, and GATA4) described above had an impact on overall survival of OSCC patients, TCGA DNA methylation data (CpG sites) with clinical information $(n=344)$ were divided into two groups (hypermethylated group, $n=172$; hypomethylated group, $n=172$ ) according to their methylation status on TFPI2, SOX17, or GATA4. Kaplan-Meier analysis was performed on either each $\mathrm{CpG}$ on the promoter region or combinations of CpGs. To explore the potential clinical significance of these data, we tested these above three tumor suppressor genes for their prognostic potential in a large TCGA cohort (head and neck squamous cell carcinoma; HNSCC) [15]. We subtracted the patients with OSCC from HNSCC TCGA cohort and well-annotated clinical data that could be correlated with survival and gene methylation status. Strikingly, we observed a statistically significant increased risk for mortality when either individual genes or combination of genes were methylated. For example, Kaplan-Meier survival curves for GATA4 and SOX17, individually, show that DNA methylation of each is associated with decreased survival but no significant statistical results for TFPI2 alone (Fig. 6a, $p=0.016$ for GATA4, $p=0.0066$ for SOX17), even though TPFI2 gene was frequently hypermethylated in both OSCC cells and OSCC primary tumors. We wondered whether the combination of twoor three-gene methylation could have an impact on overall survival in this cohort. Very interestingly, 


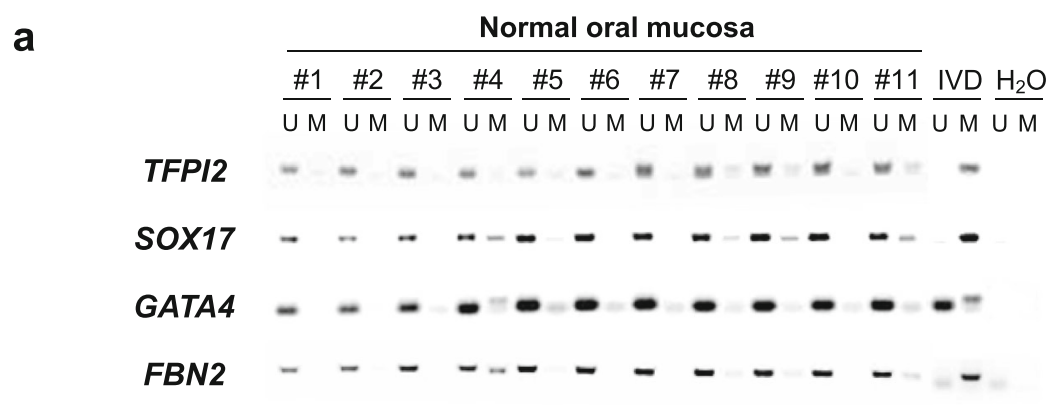

b

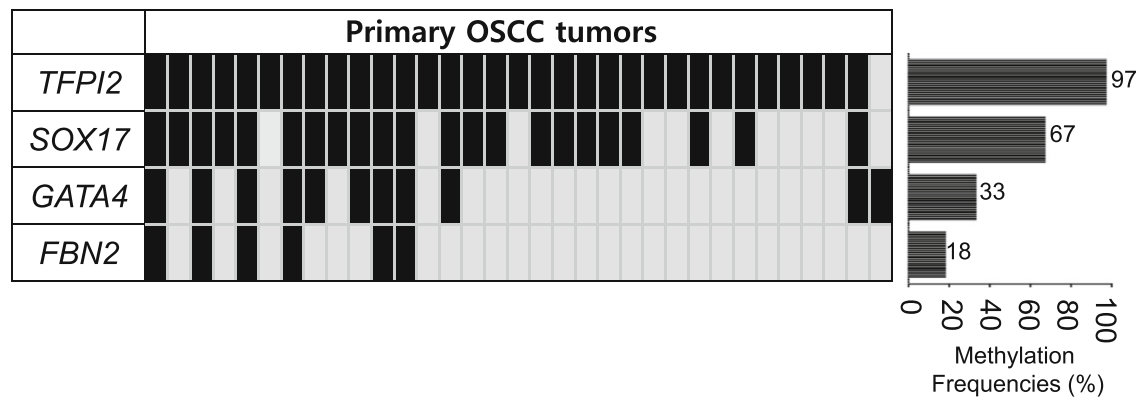

Fig. 3 Epigenetic inactivation of TFPI2, SOX17, GATA4, and FBN2 in normal oral mucosa and OSCC primary tissues. a Methylation status of TFPI2, SOX17, GATA4, and FBN2 in normal oral mucosa $(n=11)$ and $\mathbf{b}$ the methylation frequency of the four genes in OSCC primary tissues $(n=33)$. $M=$ methylation signal; $\mathrm{U}=$ unmethylated signal; IVD = in vitro methylated $\mathrm{DNA} ; \mathrm{dd} \mathrm{H}_{2} \mathrm{O}=$ water control without DNA. The bar graph shows the MSP results of all primary samples

combination of two-gene (SOX17 and GATA4) methylation is associated with significantly worse survival (Fig. 6b, $p=0.013$ ). In addition, SOX17 combination of TFPI2 gene methylation is associated with worse survival as well (Fig, 6)b, $p=0.019$, but there are no statistically significant results for combination of GATA4 and TFPI2 genes. More importantly, Kaplan-Meier survival curves for TFPI2, GATA4, and SOX17 show that DNA methylation of three-gene combination is associated with significantly worse survival (Fig. $6 c, p=0.0041$ ). These findings support our hypothesis that DNA methylation of TSGs may have an important role during OSCC cancer progression as well as clinical application as prognostic biomarkers.

\section{Discussion}

The aberrant promoter hypermethylation in the adjacent of transcription start sites (TSSs) often leads to alterations in gene function and abnormal cellular pathway in human cancer. Epigenetic events linked to TSG inactivation through promoter methylation are as frequent as somatic mutations in cancer and contribute to drive tumor initiation and progression. The promoter methylation of 14 selected TSGs in this study has previously been identified in colon cancer $[3,16]$ and has been characterized in other cancer types [4]. Recently, it has been demonstrated that
OSCC involves genetic alterations and epigenetic mechanisms play crucial roles [17]. Epigenetically regulated genes have become an important tool for better understanding cancer initiation, progression, and development.

Interestingly, recent studies have demonstrated that Fusobacterium nucleatum (Fn), a specific species of the gut microbiota, may be closely associated with colorectal carcinogenesis [18]. A growing body of evidence suggests that Fn promotes tumor progression and inhibits the antitumor immune response in the colorectum by regulating the $\beta$-catenin pathway $[8,18]$. In addition, Fn DNA is significantly enriched in the microsatellite instability-high (MSI-H) molecular subtypes of CRC samples $[19,20]$. These studies encouraged us to investigate whether the epigenome profile of colon cancer might be shared with OSCC. We therefore tested the hypothesis that selected TSGs, which are frequently hypermethylated in colon cancer, predominantly promote methylation in OSCC tumors. We observed interesting relationships among the most methylated genes (TFPI2, SOX17, and GATA4) in OSCC cell lines and primary tumors in a cancer-specific manner.

Tissue factor pathway inhibitor-2 (TFPI2), a Kunitztype serine proteinase inhibitor, can inhibit a variety of serine proteases, including factor VIIa/tissue factor, factor Xa, plasmin, trypsin, chymotrypsin, and plasma 


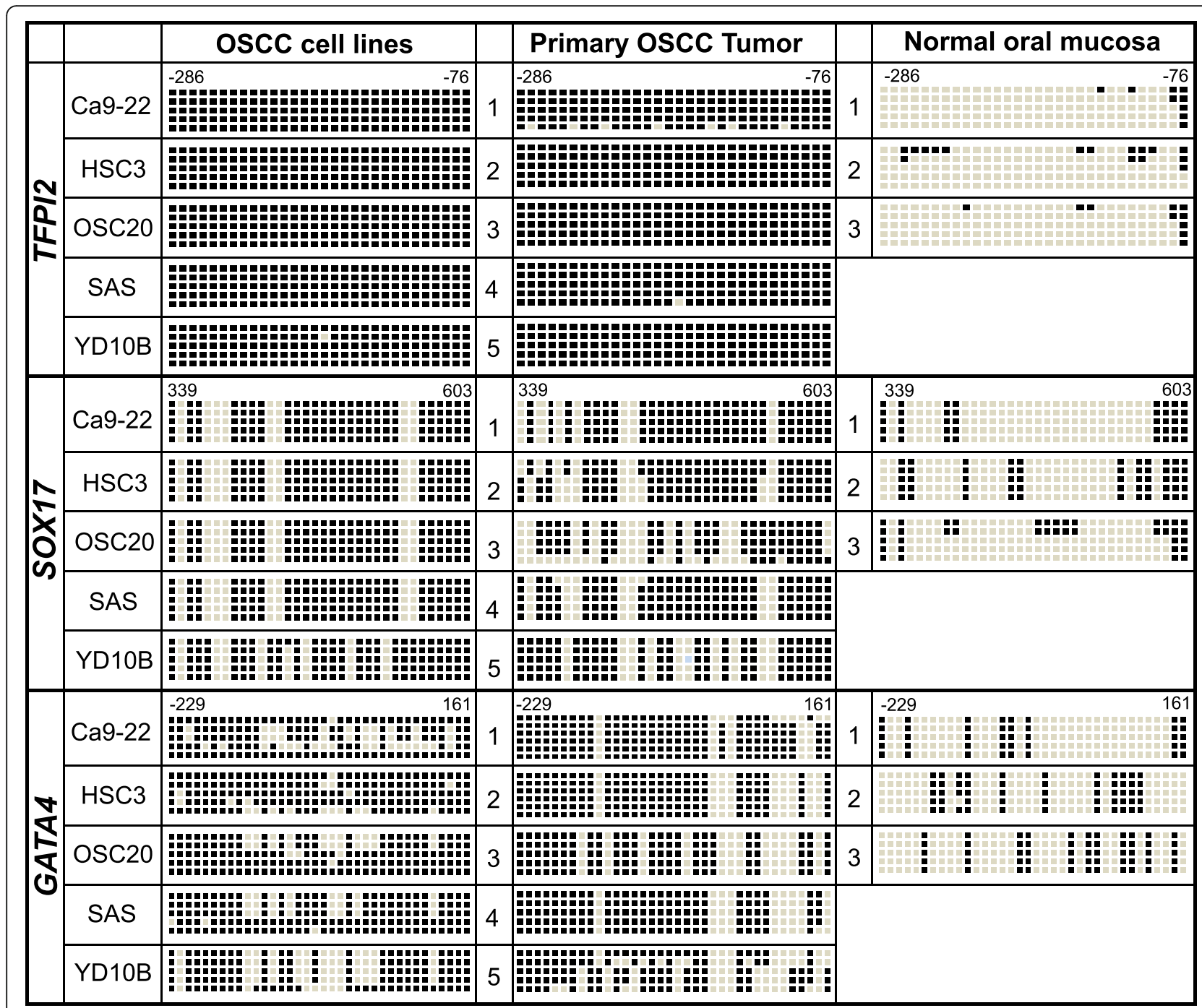

Fig. 4 Bisulfite sequencing analyses of the CPG islands in the TFPI2, SOX17, and GATA4 gene promoter regions. Representative bisulfite sequencing analyses were performed for all three genes in representative OSCC cell lines (Ca9-22, HSC3, OSC20, SAS, and YD10B), OSCC primary tumors $(n=5)$, and normal oral mucosa $(n=3)$. The location of CpG sites (TFPI2: upstream region from - 286 to -76 ; SOX17: exon 1 region from + 339 to + 603; and GATA4: upstream region from -229 to + 161) relative to the transcription start sites (TSSs) of exon 1. Each box represents a CpG dinucleotide. The black boxes represent methylated cytosines, and the white boxes represent unmethylated cytosines

kallikrein. Over the last decade, TFPI2 has been identified as a TSG in several types of cancer, including colorectal cancer (CRC) [21, 22]. TFPI2 methylation frequently existed in CRC patients' sera [23] and stool samples [21]. Moreover, hypermethylated TFPI2 was associated with recurrence and early-stage CRC [24], and TFPI2 was significant in CRC patients' sera with large, poorly differentiated carcinoma, deep invasion, lymph node metastasis, or distant metastasis [23]. Although the methylation of TFPI2 in OSCC has been identified using a genome-wide methylation array, its promoter hypermethylation associated with transcriptional silencing was not validated in OSCC tumor samples [25]. In this study, we validated that TFPI 2 is frequently methylated in a panel of OSCC primary tumor samples in a cancer-specific manner and that TFPI2 is transcriptionally regulated by promoter hypermethylation in OSCC cells. Our study thus highlights the finding that TFPI2 is hypermethylated in most OSCC tumor samples we tested in this study, suggesting that TFPI2 may be a useful biomarker for screening OSCC patients. Therefore, further studies in a larger series of samples will be necessary to confirm that the TFPI2 gene can be a useful methylation biomarker for screening OSCC patients.

SOX17 encodes an HMG box transcription factor and has been implicated in oligodendrocyte development, vascular development, the formation of definitive endoderm, and embryonic hematopoiesis [26-29]. The SOX17 

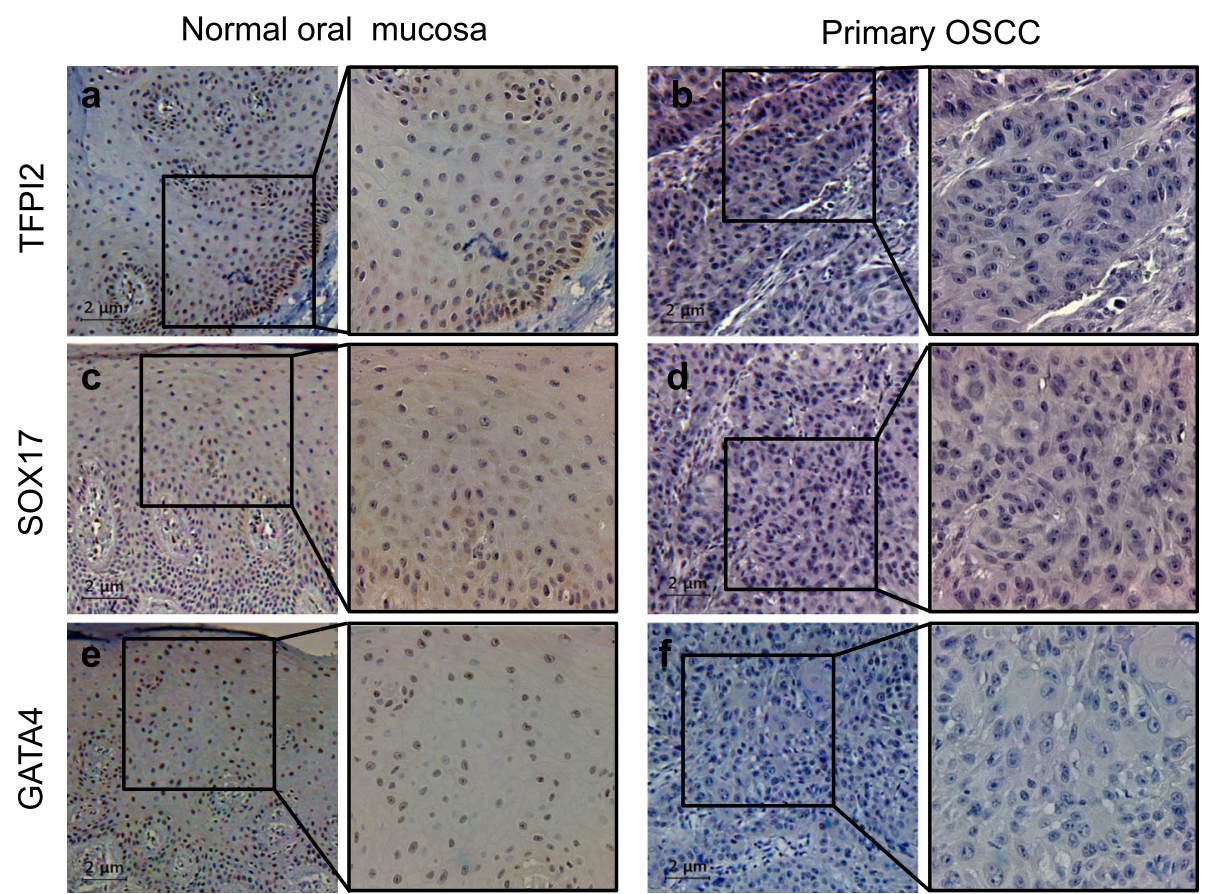

Fig. 5 Protein expression levels of TFPI2, SOX17, and GATA4 in primary OSCC tumors and normal oral mucosa. Representative immunohistochemical analysis results showing $\mathbf{a}, \mathbf{b}$ TFPI2, $\mathbf{c}$, $\mathbf{d}$ SOX17, and $\mathbf{e}, \mathbf{f}$ GATA4 expression in primary OSCC tissues and normal oral mucosa (right images: $\times 100$; left images: $\times 400$ )

promoter is hypermethylated in cholangiocarcinoma (CCA) tissues, lung cancer [30], gastric cancer [31], liver cancer [32], and breast cancer [33]. Additionally, epigenetically regulated SOX17 may contribute to the abnormal activation of the Wnt signaling pathway in human cancer [34]. The high frequency of SOX17 methylation in 50\% of non-small-cell lung cancers and nearly $90 \%$ of esophageal squamous cancers strongly supports this possibility and is consistent with the known role of the aberrant activation of Wnt signaling during tumorigenesis for multiple cancer types [35].

The GATA4 gene encodes a member of the GATA family of zinc-finger transcription factors, which recognize the GATA motif present in the promoters of many genes. Transcription factors GATA4 play an essential role in the development and differentiation of the gastrointestinal tract and are suggested to be involved in colorectal cancer development [36, 37]. GATA4 is known to be more likely to behave as a tumor suppressor gene since the increased expression levels correlate with the terminal differentiation in the intestinal epithelium and the terminal differentiation induced in CRC cells by sodium butyrate [36].

The loss of GATA4 expression due to promoter hypermethylation has been reported in primary colorectal, gastric, esophageal, lung, and ovarian cancers [37-40]. To our knowledge, this is the first study to describe how the promoter hypermethylation of both the SOX17 and GATA4 genes is regulated in OSCC cells and how these promoters are frequently hypermethylated in OSCC primary tumors.

Epigenetic alterations of specific genes have recently emerged as potential candidate biomarkers for the early detection of cancer [1]. Recent advances attest to the great promise of DNA methylation markers as powerful tools for future use in the clinic. It would be of great value to find useful biomarkers and prognostic molecular signatures in OSCC to develop novel therapeutic strategies or chemotherapeutic agents. Numerous potential clinical applications of epigenetics for diagnostic and therapeutic applications have been reported [41].

The promoter hypermethylation of specific genes has already been studied in oral premalignancy [42, 43], but little is known about the description of early DNA methylation changes during oral carcinogenesis using genome-wide profiles. Recently, several studies have reported that early DNA promoter methylation alterations are associated with histological changes during oral tumorigenesis [44]. They confirmed that these alterations are early events during oral tumorigenesis and offer an opportunity for biomarker development [45].

According to our KM analysis for patients with OSCC from TCGA data, we found a statistically significant increased risk for mortality when either individual genes 

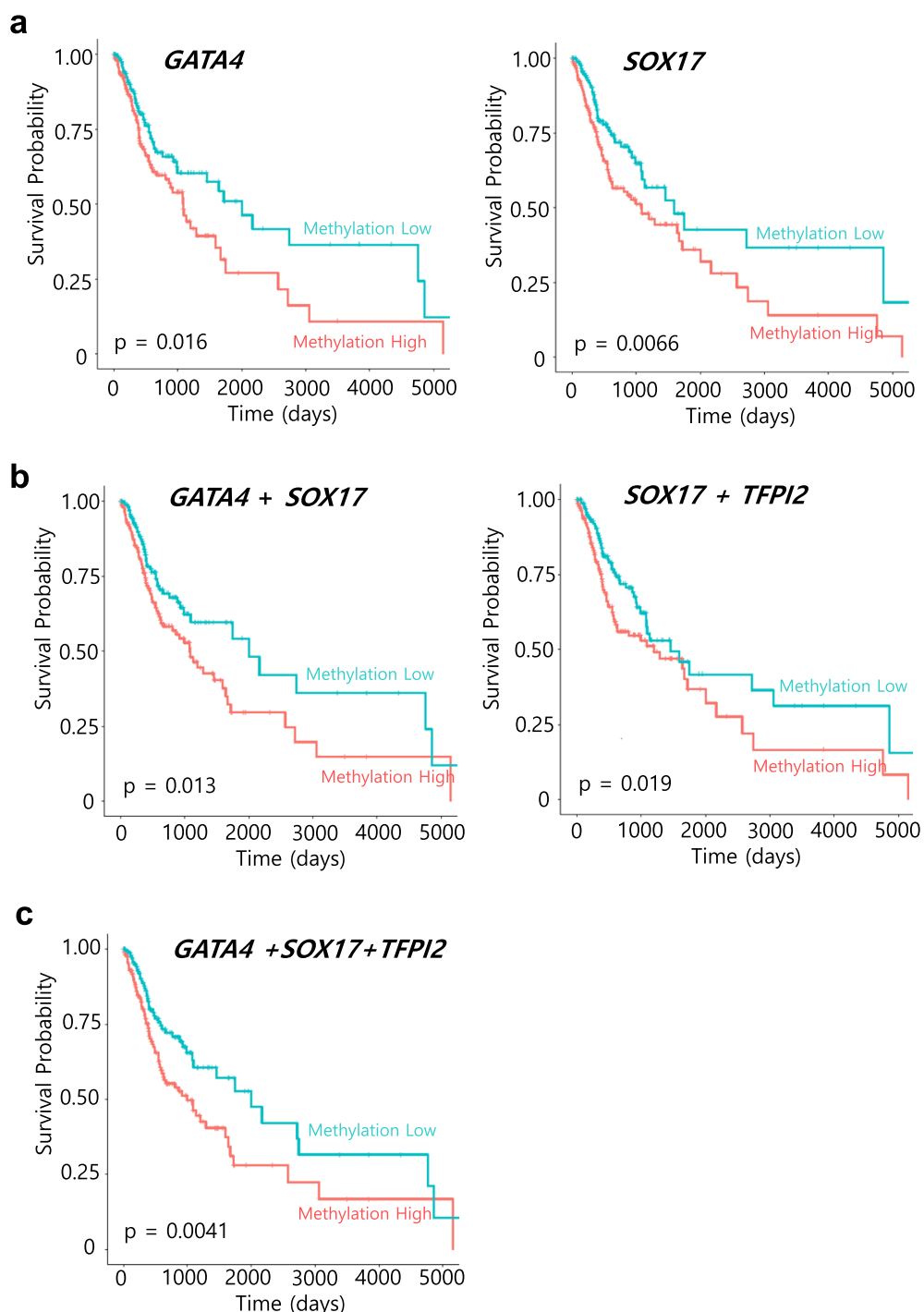

Fig. 6 Kaplan-Meier survival curves for the 344 patients with OSCC from TCGA HNSCC cohort according to the methylation status of the three candidate genes. Overall survival for a GATA4 (cg24910352 located on gene body) and SOX17 (cg04672706 located on promoter region), respectively. b Combination of GATA4 and SOX17 genes methylation (left) and TFPI2 (cg22441533 located on promoter region) and SOX17 genes methylation (right). c Combination of TFPI2, GATA4, and SOX17 genes methylation. Probe positions were annotated using the UCSC genome browser. Clinical information of patients with OSCC $(n=344)$ were divided into two groups (methylation high group; orange line, $n=172$; methylation low group; blue line, $n=172$ ) according to their methylation status ( $\beta$-values) on TFP12, SOX17, or GATA4. A probability of $<0.05$ (log rank test ${ }^{*} p<0.05$ ) was considered to represent a statistically significant difference

or combination of genes were methylated. Unlikely SOX17 and GATA4, we did not see any significant clinical correlation with overall survival of TFPI2 alone. Promoter methylation of TFPI2 is detected in most of tumors (over 90\% tested cancer patients samples) with OSCC and other cancer types [21] as well which explained TFPI 2 methylation can be useful for early detection biomarker rather than prognostic biomarkers. Previous studies have reported survival-related OSCC methylation biomarkers [46, 47]. These reports described novel methylation biomarkers associated with prognostic biomarkers in OSCC, showing that the methylation frequency of candidate genes ranged from 10 to $65 \%$. However, TFPI2 and SOX17 in our study showed a significant hypermethylation pattern $(95 \%$ and $67 \%$, respectively) in OSCC primary tumors compared to those observed in previous studies because we used a well-established validation approach using a specific promoter region to identify methylation biomarker candidates. Based on our data, the dysregulation of the TFPI2, 
SOX17, and GATA4 genes regulated by epigenetic changes may explain a main mechanism linked to OSCC development, and these three-gene panels could serve as synergistic prognostic biomarkers in OSCC treatment. Further studies are needed to verify TFPI2 and SOX17 as methylation biomarkers in a large cohort of OSCC primary tumors along with normal controls, and these genes should be evaluated by testing in blood or sputum DNA.

\section{Conclusions}

In summary, we used 14 putative TSGs originally identified in colon cancer to screen the epigenetic regulation of these genes in 10 OSCC cell lines, a small set of primary OSCC tumors, and normal oral mucosa. We identified the transcriptional expression of 9 genes regulated by promoter hypermethylation in OSCC and finally provided 3 genes (TFPI2, SOX17, and GATA4) that were frequently hypermethylated in primary OSCC tumors in a cancer-specific manner. The methylation level in the promoter region of these genes was confirmed by bisulfite genomic sequencing analysis, which suggested that the methylation level is significantly increased in OSCC cell lines and primary tumors compared to the methylation level in normal tissues. Our study thus highlights the finding that the dysregulation of TFPI2, SOX17, and GATA4 genes regulated by epigenetic changes may explain a main mechanism linked to OSCC development, and this three-gene panel has a potential to be used as a synergistic biomarker set capable of improving the prognosis and treatment for patients with OSCC.

\section{Methods}

\section{Tissue samples}

For OSCC samples, paraffin-embedded tissue specimens from patients who were diagnosed and surgically treated at the Department of Oral and Maxillofacial Surgery, Pusan National University Dental Hospital, between 2009 and 2014 were retrieved. For normal oral mucosal tissue for comparison, fresh healthy tissue that had been obtained during dental procedures, such as gingivoplasty, third molar extraction, or implant surgery, was used. Clinicopathologic features, including age, gender, histopathologic grade, and the TNM stage, are summarized in Table 1. Tumor grading was based on the ethical permission for this study and was granted by the Institutional Review Board (IRB) of Pusan National University Dental Hospital (IRB No. PNUDH-2019-006).

\section{Cell culture and 5-aza-dC treatment}

Ten human OSCC cell lines (Ca9-22, HSC4, OSC20, SAS, SCC25, HN22, YD10B, YD38, YD9, and HSC3) were used in this study. The Ca9-22 and HSC4 cell lines were cultured in MEM/EBSS (HyClone, Logan, UT) medium. The
OSC20, SAS, and SCC25 cell lines were cultured in a 1:1 mixture of Dulbecco's modified Eagle's medium and Ham's F-12 Nutrient Mixture (DMEM/F12; HyClone, Logan, UT). The HN22, YD10B, YD38, YD9, and HSC3 cell lines were cultured in a 1:1 mixture of Dulbecco's modified Eagle's medium (HyClone, Logan, UT). All cell culture medium was supplemented with $10 \%$ fetal bovine serum (HyClone, Logan, UT, USA) and 1\% antibioticantimycotic (Gibco, Grand Island, NY, USA). All cell lines were incubated at $37^{\circ} \mathrm{C}$ in atmospheric conditions of $20 \%$ $\mathrm{O}_{2}$ and $5 \% \mathrm{CO}_{2}$. To investigate the effects of 5-aza-dC treatment, the cells were treated with $5 \mu \mathrm{M} 5$-aza-dC (Sigma, St. Louis, MO, USA) for $72 \mathrm{~h}$.

\section{DNA methylation analysis}

For methylation analyses, genomic DNA was isolated from 10 OSCC cell lines, 33 primary OSCC tissues, and 11 normal oral mucosa samples using phenol/chloroform. The bisulfite modification of $2 \mu \mathrm{g}$ genomic DNA was performed using the EZ DNA Methylation Kit (Zymo Research, CA, USA). For positive and negative controls, in vitro methylated DNA (IVD) and $\mathrm{H}_{2} \mathrm{O}$ were used. Primer pairs for methylation analyses were preferentially designed for CpG islands of the target genes [21, 34, 37, 48]. The primer sequences are listed in Additional file 2: Table S1. Methylation analyses, including MSP and bisulfite sequencing analysis, were performed as previously described [49].

\section{Bisulfite sequencing}

One microgram of genomic DNA from each sample was bisulfite converted using the EZ DNA Methylation Kit (Zymo Research, CA, USA) following the manufacturer's protocol. The PCR conditions and primer sequences are provided in Additional file 2: Table S1. The PCR amplicons were gel-purified and subcloned into the pCRIITOPO vector (Invitrogen, Carlsbad, CA). At least five to seven clones were randomly selected and sequenced on an ABI3730xl DNA analyzer to ascertain the methylation patterns of each locus.

\section{Quantitative real-time RT-PCR}

Total RNA was isolated from the human normal colon and UC patient tissues using TRI-Solution (Bio Science Technology) following the manufacturer's protocol. RNA quantity was measured using a NanoDrop 2000/2000c instrument (Thermo Scientific, MA, USA), and $1 \mu \mathrm{g}$ of total RNA was reverse-transcribed into cDNA using the iScript ${ }^{\mathrm{TM}}{ }^{\mathrm{CDNA}}$ Synthesis Kit (Bio-Rad). For expression studies, the primers are listed in Additional file 2: Table S1. Quantitative RT-PCR was performed on a CFX96 ${ }^{\mathrm{TM}}$ Real-Time PCR Detection System (Bio-Rad) using SYBR Green Master Mix (Thermo Scientific, MA, USA). The expression levels of the target genes were normalized to 
the GAPDH levels, and all the relative quantifications of expression were calculated using the $2^{-\Delta \Delta \mathrm{Ct}}$ method.

\section{Immunohistochemistry (IHC)}

Formalin-fixed, paraffin-embedded specimens of OSCC and normal oral mucosal tissues were sectioned to a 5$\mu \mathrm{m}$ thickness. The sections were deparaffinized in xylene and rehydrated through graded alcohol solutions. Heatinduced antigen retrieval was performed in a microwave oven for $10 \mathrm{~min}$ in Tris/EDTA buffer ( $\mathrm{pH}$ 9.0). Endogenous peroxidase activity was inactivated by treating with $3 \% \mathrm{H}_{2} \mathrm{O}_{2}$ in $\mathrm{PBS}$ for $15 \mathrm{~min}$. After blocking nonspecific binding $(0.75 \%$ BSA in PBS), the sections were incubated with the anti-TFPI2 (Abcam, dilution 1:100), antiSOX17 (Abcam, dilution 1:100), and anti-GATA4 antibodies (Abcam, dilution 1:100) overnight at $4{ }^{\circ} \mathrm{C}$. The staining was visualized by a peroxidase-conjugated secondary antibody and diaminobenzidine (Vector labs, Burlingame, CA, USA). Finally, the sections were counterstained by Mayer's hematoxylin and were mounted and photographed with an Axioplan microscope (Carl Zeiss, Germany). Primary antibodies were omitted for the negative controls.

\section{Kaplan-Meier survival analysis using TCGA DNA methylation data}

Head and neck squamous cell carcinoma (HNSCC) DNA methylation dataset (HumanMethylation $450 \mathrm{k}$ Illumina; $n=528$ ) in The Cancer Genome Atlas (TCGA) database [15] was initially downloaded from the firebrowse website (http://firebrowse.org/). Then, samples related to OSCC $(n=344)$ were selected for survival analysis from HNSCC TCGA cohort (patients $(n=184)$ from tonsil, larynx, oropharynx, hypopharynx, and lip were excluded based on anatomic subdivision). Patients were divided into two groups (high-hypermethylated, $n=174$; low-hypomethylated, $n=174)$ according to the methylation level ( $\beta$ value) on each CpG site located in the promoter regions of TFPI2 and SOX17, but in gene body region of GATA4. The $\mathrm{R}$ package survminer (https://github.com/kassam bara/survminer) was used for the analysis. $P$ values were calculated using the log-rank test.

\section{Statistical analysis}

Quantified data are expressed as the mean \pm standard deviation (SD). Significance testing was conducted using Student's $t$ test.

\section{Additional files}

Additional file 1: Figure S1. Correlation between transcriptional expression by qRT-PCR and the promoter hypermethylation of tumor suppressor genes by MSP analysis in OSCC cell lines. Related to Fig. 1 a. Figure S2. Protein expression levels of TFPI2, SOX17, and GATA4 in additional set of primary OSCC tumors and normal oral mucosa. Related to Fig. 5. (PPTM 3917 kb)

Additional file 2: Table S1. Selected gene primers for MSP, bisulfite sequencing, and RT-PCR analyses. (DOCX $13 \mathrm{~kb}$ )

\section{Abbreviations}

5-aza-dC: 5-Aza-2'-deoxycytidine; Fn: Fusobacterium nucleatum; IHC: Immunohistochemistry; KM survival curve: Kaplan-Meier survival curves; MSP: Methylation-specific PCR; OSCC: Oral squamous cell carcinoma; RT-PCR: Reverse transcriptase polymerase chain reaction; TSGs: Tumor suppressor genes; TSSs: Transcription start sites

\section{Acknowledgements}

We would like to thank Prof. Khadijah A. Mitchell of the Lafayette College for the critical reading of the manuscript and providing language editing.

\section{Authors' contributions}

JMY and HRP contributed to the study design. SYK, YKH, and JMY performed the experiments and generated the data. $\mathrm{CHL}$ and JMS generated the clinical data. KK analyzed the clinical data using TCGA DNA methylation dataset. JMY and HRP wrote the manuscript. All authors contributed to the critical revision of the final manuscript and approved the final version of the manuscript.

\section{Funding}

This work was supported by the National Research Foundation of Korea (NRF) grant funded by the Korean Government (MSIT) (No. NRF-2018R1A5A2023879 and No. NRF-2017R1A2B4005588).

Availability of data and materials

Not applicable.

Ethics approval and consent to participate

This study was approved by the Institutional Review Board of Pusan National University Dental Hospital (IRB No. PNUDH-2019-006), and written, informed consent was obtained for all study participants prior to data collection.

Consent for publication

Not applicable.

\section{Competing interests}

The authors declare that they have no competing interests.

\section{Author details}

${ }^{1}$ Department of Oral Pathology, School of Dentistry, Pusan National University, Yangsan 50612, Gyeongsangnam-do, Republic of Korea. ${ }^{2}$ Department of Microbiology and Immunology, College of Medicine, Inje University, Busan 47392, Republic of Korea. ${ }^{3}$ Department of Oral and Maxillofacial Surgery, Pusan National University, Yangsan 50612, Gyeongsangnam-do, Republic of Korea. ${ }^{4}$ Dental and Life Science Institute, School of Dentistry, Pusan National University, Yangsan 50612, Gyeongsangnam-do, Republic of Korea. ${ }^{5}$ Department of Pathology, School of Medicine, Pusan National University, Busan 49241, Republic of Korea.

${ }^{6}$ Department of Microbiology, Dankook University, Cheonan 31116, Republic of Korea.

Received: 11 June 2019 Accepted: 22 July 2019

Published online: 12 August 2019

\section{References}

1. Jones PA, Baylin SB. The epigenomics of cancer. Cell. 2007;128(4):683-92.

2. Herman JG, Baylin SB. Gene silencing in cancer in association with promoter hypermethylation. N Engl J Med. 2003;349(21):2042-54.

3. Schuebel KE, Chen W, Cope L, Glöckner SC, Suzuki H, Yi JM, et al. Comparing the DNA hypermethylome with gene mutations in human colorectal cancer. PLoS Genetics. 2007;3(9):1709-23.

4. Esteller M. Epigenetic gene silencing in cancer: the DNA hypermethylome. Hum Mol Genet. 2007;16:R50-9. 
5. Shen L, Kondo Y, Guo Y, Zhang J, Zhang L, Ahmed S, et al. Genome-wide profiling of DNA methylation reveals a class of normally methylated CpG island promoters. PLOS Genetics. 2007;3(10):e181.

6. Heyn H, Esteller M. DNA methylation profiling in the clinic: applications and challenges. Nat Rev Genet. 2012;13(10):679-92.

7. Castellarin M, Warren RL, Freeman JD, Dreolini L, Krzywinski M, Strauss J, et al. Fusobacterium nucleatum infection is prevalent in human colorectal carcinoma. Genome Res. 2012;22(2):299-306.

8. Kostic Aleksandar D, Chun E, Robertson L, Glickman Jonathan N, Gallini Carey A, et al. Fusobacterium nucleatum potentiates intestinal tumorigenesis and modulates the tumor-immune microenvironment. Cell Host Microbe. 2013;14(2):207-15.

9. Marur S, Forastiere AA. Head and neck squamous cell carcinoma: update on epidemiology, diagnosis, and treatment. Mayo Clin Proc. 2016;91(3):386-96.

10. Argiris A, Karamouzis MV, Raben D, Ferris RL. Head and neck cancer. Lancet. 2008;371(9625):1695-709.

11. Scully C, Bagan J. Oral squamous cell carcinoma overview. Oral Oncol. 2009; 45(4):301-8.

12. Scully C, Bagan JV. Recent advances in Oral Oncology 2007: Imaging, treatment and treatment outcomes. Oral Oncol. 2008;44(3):211-5.

13. Pitiyage G, Tilakaratne WM, Tavassoli M, Warnakulasuriya S. Molecular markers in oral epithelial dysplasia: review. J Oral Pathol Med. 2009;38(10):737-52.

14. Choi S, Myers JN. Molecular pathogenesis of oral squamous cell carcinoma: implications for therapy. J Dent Res. 2008;87(1):14-32.

15. The Cancer Genome Atlas. Comprehensive genomic characterization of head and neck squamous cell carcinomas. Nature. 2015;517:576.

16. Suzuki H, Watkins DN, Jair KW, Schuebel KE, Markowitz SD, Chen WD, et al. Epigenetic inactivation of SFRP genes allows constitutive WNT signaling in colorectal cancer. Nat Genet. 2004;36(4):417-22.

17. Jithesh PV, Risk JM, Schache AG, Dhanda J, Lane B, Liloglou T, Shaw RJ. The epigenetic landscape of oral squamous cell carcinoma. Br J Cancer. 2013; 108:370

18. Flemer $\mathrm{B}$, Warren RD, Barrett MP, et al. The oral microbiota in colorectal cancer is distinctive and predictive. Gut. 2018;67:1454-63.

19. Tahara T, Yamamoto E, Suzuki H, Maruyama R, Chung W, Garriga J, et al. Fusobacterium in colonic flora and molecular features of colorectal carcinoma. Cancer Res. 2014;74(5):1311.

20. Mima K, Nishihara R, Qian ZR, Cao Y, Sukawa Y, Nowak JA, et al. Fusobacterium nucleatum in colorectal carcinoma tissue and patient prognosis. Gut. 2016;65(12):1973.

21. Glockner SC, Dhir M, Yi JM, McGarvey KE, Van Neste L, Louwagie J, et al. Methylation of TFPI2 in stool DNA: a potential novel biomarker for the detection of colorectal cancer. Cancer Res. 2009;69(11):4691-9.

22. Liu Z, Zhang J, Gao Y, Pei L, Zhou J, Gu L, et al. Large-scale characterization of DNA methylation changes in human gastric carcinomas with and without metastasis. Clin Cancer Res. 2014;20(17):4598.

23. Hibi K, Goto T, Shirahata A, Saito M, Kigawa G, Nemoto H, et al. Detection of TFPI2 methylation in the serum of colorectal cancer patients. Cancer Lett. 2011;311(1):96-100.

24. Rasmussen $\mathrm{SL}$, Krarup HB, Sunesen $\mathrm{KG}$, Pedersen IS, Madsen PH, Thorlacius-Ussing O. Hypermethylated DNA as a biomarker for colorectal cancer: a systematic review. Color Dis. 2016;18(6):549-61.

25. Li Y-F, Hsiao Y-H, Lai Y-H, Chen Y-C, Chen Y-J, Chou J-L, et al. DNA methylation profiles and biomarkers of oral squamous cell carcinoma. Epigenetics. 2015;10(3):229-36.

26. Sohn J, Natale J, Chew L-J, Belachew S, Cheng Y, Aguirre A, et al. Identification of SOX17 as a transcription factor that regulates oligodendrocyte development. J Neurosci. 2006;26(38):9722.

27. Matsui T, Kanai-Azuma M, Hara K, Matoba S, Hiramatsu R, Kawakami H, et al. Redundant roles of SOX17 and Sox18 in postnatal angiogenesis in mice. J Cell Sci. 2006;119(17):3513.

28. Park K-S, Wells JM, Zorn AM, Wert SE, Whitsett JA. SOX17 influences the differentiation of respiratory epithelial cells. Dev Biol. 2006;294(1): 192-202.

29. Kim I, Saunders TL, Morrison SJ. SOX17 dependence distinguishes the transcriptional regulation of fetal from adult hematopoietic stem cells. Cell. 2007;130(3):470-83.

30. Hulbert A, Jusue-Torres I, Stark A, Chen C, Rodgers K, Lee B, et al. Early detection of lung cancer using DNA promoter hypermethylation in plasma and sputum. Clin Cancer Res. 2017;23(8):1998.
31. Oishi Y, Watanabe Y, Yoshida Y, Sato Y, Hiraishi T, Oikawa R, et al. Hypermethylation of SOX17 gene is useful as a molecular diagnostic application in early gastric cancer. Tumor Biol. 2012;33(2):383-93.

32. Jia Y, Yang Y, Liu S, Liu S, Herman JG, Lu F, et al. SOX17 antagonizes WNT/ $\beta$-catenin signaling pathway in hepatocellular carcinoma. Epigenetics. 2010; 5(8):743-9.

33. Fu D-Y, Wang Z-M, Li C, Wang B-L, Shen Z-Z, Huang W, et al. SOX17, the canonical Wnt antagonist, is epigenetically inactivated by promoter methylation in human breast cancer. Breast Cancer Res Treat. 2010;119(3): 601-12.

34. Zhang W, Glockner SC, Guo M, Machida M, Ota E, Wang DH, et al. Epigenetic inactivation of the canonical Wnt antagonist SRY-box containing gene 17 in colorectal cancer. Cancer Res. 2008;68(8):2764-72.

35. Clevers $H$. Wnt/ $\beta$-catenin signaling in development and disease. Cell. 2006; 127(3):469-80.

36. Gao $X$, Sedgwick T, Shi YB, Evans T. Distinct functions are implicated for the GATA-4, -5 , and -6 transcription factors in the regulation of intestine epithelial cell differentiation. Mol Cell Biol. 1998;18(5):2901-11.

37. Akiyama Y, Watkins $N$, Suzuki H, Jair KW, van Engeland M, Esteller M, et al. GATA-4 and GATA-5 transcription factor genes and potential downstream antitumor target genes are epigenetically silenced in colorectal and gastric cancer. Mol Cell Biol. 2003:23(23):8429-39.

38. Guo M, Akiyama Y, House MG, Hooker CM, Heath E, Gabrielson E, et al. Hypermethylation of the GATA genes in lung cancer. Clin Cancer Res. 2004; 10(23):7917-24.

39. Guo M, House MG, Akiyama Y, Qi Y, Capagna D, Harmon J, Baylin SB, Brock MV, Herman JG. Hypermethylation of the GATA gene family in esophageal cancer. Int J Cancer. 2006;119(9):2078-83.

40. Wakana K, Akiyama Y, Aso T, Yuasa Y. Involvement of GATA-4/-5 transcription factors in ovarian carcinogenesis. Cancer Lett. 2006; 241(2):281-8.

41. Laird PW. The power and the promise of DNA methylation markers. Nat Rev Cancer. 2003:3(4):253-66.

42. Pattani KM, Zhang Z, Demokan S, Glazer C, Loyo M, Goodman S, et al. Independently associated with risk of oral cavity cancer and premalignancy. Cancer Prev Res. 2010;3(9):1093.

43. Schussel J, Zhou XC, Zhang Z, Pattani K, Bermudez F, Jean-Charles G, et al. EDNRB and DCC salivary rinse hypermethylation has a similar performance as expert clinical examination in discrimination of oral cancer/dysplasia versus benign lesions. Clin Cancer Res. 2013;19(12):3268.

44. Towle R, Truong D, Hogg K, Robinson WP, Poh CF, Garnis C. Global analysis of DNA methylation changes during progression of oral cancer. Oral Oncol. 2013;49(11):1033-42.

45. Arantes LMRB, de Carvalho AC, Melendez ME, Carvalho AL, Goloni-Bertollo EM. Methylation as a biomarker for head and neck cancer. Oral Oncol. 2014; 50(6):587-92.

46. Foy J-P, Pickering CR, Papadimitrakopoulou VA, Jelinek J, Lin SH, William $W N$, et al. New DNA methylation markers and global DNA hypomethylation are associated with oral cancer development. Cancer Prev Res. 2015:8(11):1027.

47. Langevin SM, Butler RA, Eliot M, Pawlita M, Maccani JZJ, McClean MD, et al. Novel DNA methylation targets in oral rinse samples predict survival of patients with oral squamous cell carcinoma. Oral Oncol. 2014;50(11):1072-80.

48. Yi JM, Dhir M, Guzzetta AA, lacobuzio-Donahue CA, Heo K, Yang KM, et al. DNA methylation biomarker candidates for early detection of colon cancer. Tumor Biol. 2012:33(2):363-72.

49. Kim J-G, Kim T-O, Bae J-H, Shim J-W, Kang MJ, Yang K, et al. Epigenetically regulated MIR941 and MIR1247 target gastric cancer cell growth and migration. Epigenetics. 2014;9(7):1018-30.

\section{Publisher's Note}

Springer Nature remains neutral with regard to jurisdictional claims in published maps and institutional affiliations. 